\title{
Hsp as a long-term buffer of the genome-wide mutation burden
}

\author{
A. Sokol ${ }^{1 *}$, K. Ushakova ${ }^{1}$, A.A. Mikhaylova ${ }^{1}$, A.G. Mikhaylova ${ }^{1}$, D. Knorre ${ }^{3}$, \\ I. Mazunin ${ }^{1}$, A. Reymond ${ }^{5}$, K. Gunbin ${ }^{1,4}$, K. Popadin ${ }^{1,5 * *}$ \\ ${ }^{1}$ The School of Life Sciences, Immanuel Kant Baltic Federal University, Kaliningrad, Russia \\ ${ }^{2}$ Institute of Biodiversity Animal Health and Comparative Medicine, University of Glasgow, Glasgow, UK \\ ${ }^{3}$ The A.N. Belozersky Institute of Physico-Chemical Biology, MSU, Moscow, Russia \\ ${ }^{4}$ The Institute of Cytology and Genetics SB RAS, Novosibirsk, Russia \\ ${ }^{5}$ Center for Integrative Genomics, University of Lausanne, Lausanne, Switzerland \\ *anastasia3sokol@yandex.ru,**konstantinpopadin@gmail.com
}

Key words: hsp90, gene expression level, effective population size

Motivation and Aim: At short evolutionary times, the expression level of hsp90 works as a buffer, i.e. strongly influences the manifestation of slightly deleterious nonsynonymous variants (SDNVs) in populations of model organisms (Drosophila, Arabidopsis, etc.) and human. The increased expression level of hsp90 compensates the burden of SDNVs by correct folding of numerous proteins. Oppositely, the decreased expression level of hsp90 can not maintain correct folding of these proteins, uncovering previously hidden burden of SDNVs. We hypothesized that the expression level of hsp90 may play an important role also at long evolutionary periods compensating genome-wide burden of slightly deleterious variants. Taking into account that the number of fixed slightly deleterious variants is higher in species with low effective population size, we expect that the expression level of hsp90 in these species is increased to partially compensate the high burden of SDNVs.

Methods and Algorithms: In this project using comparative-species approach we test our hypothesis on three different levels: (i) comparison of the expression level of hsp90 between different species and tissues, (ii) investigation of the patterns of molecular evolution of hsp90 between species and (iii) analysis of the number of copies of the hsp90 genes in different species.

Results: We derived hsp90 data-base, containing the expression levels, patterns of molecular evolution and copy numbers of hsp90 genes in all eukaryotes.

Conclusion: The database will allow us to address our questions and test the hypothesis. Acknowledgements: The study was supported by the 5 Top 100 Russian Academic Excellence Project at the Immanuel Kant Baltic Federal University. 